PROCEEDINGS OF THE

AMERICAN MATHEMATICAL SOCIETY

Volume 137, Number 4, April 2009, Pages 1405-1410

S 0002-9939(08)09755-4

Article electronically published on November 5, 2008

\title{
TOTALLY FREE ARRANGEMENTS OF HYPERPLANES
}

\author{
TAKURO ABE, HIROAKI TERAO, AND MASAHIKO YOSHINAGA
}

(Communicated by Martin Lorenz)

\begin{abstract}
A central arrangement $\mathcal{A}$ of hyperplanes in an $\ell$-dimensional vector space $V$ is said to be totally free if a multiarrangement $(\mathcal{A}, m)$ is free for any multiplicity $m: \mathcal{A} \rightarrow \mathbb{Z}_{>0}$. It has been known that $\mathcal{A}$ is totally free whenever $\ell \leq 2$. In this article, we will prove that there does not exist any totally free arrangement other than the obvious ones, that is, a product of one-dimensional arrangements and two-dimensional ones.
\end{abstract}

\section{INTRODUCTION}

Let $V$ be an $\ell$-dimensional vector space $(\ell \geq 1)$ over $\mathbb{K}$ with a coordinate system $\left\{x_{1}, \ldots, x_{\ell}\right\} \subset V^{*}$. Define $S:=\operatorname{Sym}\left(V^{*}\right) \simeq \mathbb{K}\left[x_{1}, \ldots, x_{\ell}\right]$. Let $\operatorname{Der}_{\mathbb{K}}(S)$ be the set of all $\mathbb{K}$-linear derivations of $S$ to itself. Then $\operatorname{Der}_{\mathbb{K}}(S)=\bigoplus_{i=1}^{\ell} S \cdot \partial_{x_{i}}$ is a free $S$ module of rank $\ell$. A central arrangement (of hyperplanes) in $V$ is a finite collection of linear hyperplanes in $V$. In this article we assume that every arrangement is central unless otherwise specified. A multiplicity $m$ is a function $m: \mathcal{A} \rightarrow \mathbb{Z}_{>0}$ and a pair $(\mathcal{A}, m)$ is called a multiarrangement. Fix a linear form $\alpha_{H}(H \in \mathcal{A})$ in such a way that $\operatorname{ker}\left(\alpha_{H}\right)=H$. The logarithmic derivation module $D(\mathcal{A}, m)$ associated with $(\mathcal{A}, m)$ is defined by

$$
D(\mathcal{A}, m):=\left\{\theta \in \operatorname{Der}_{\mathbb{R}}(S) \mid \theta\left(\alpha_{H}\right) \in S \cdot \alpha_{H}^{m(H)} \text { for all } H \in \mathcal{A}\right\} .
$$

In general, $D(\mathcal{A}, m)$ is not necessarily a free $S$-module. We say that $(\mathcal{A}, m)$ is free if $D(\mathcal{A}, m)$ is a free $S$-module. For a fixed arrangement $\mathcal{A}$, a multiplicity $m$ on $\mathcal{A}$ is called free if a multiarrangement $(\mathcal{A}, m)$ is free. Define

$$
\mathcal{N} \mathcal{F} \mathcal{M}(\mathcal{A}):=\left\{m: \mathcal{A} \rightarrow \mathbb{Z}_{>0} \mid m \text { is not a free multiplicity }\right\} .
$$

The following definition was introduced in [4, Definition 5.4].

Definition 1.1. An arrangement $\mathcal{A}$ is called totally free if every multiplicity $m$ : $\mathcal{A} \rightarrow \mathbb{Z}_{>0}$ is a free multiplicity, or equivalently $\mathcal{N} \mathcal{F} \mathcal{M}(\mathcal{A})=\emptyset$.

When $\mathcal{A}_{i}$ is an arrangement in $V_{i}(i=1,2)$, the product $\mathcal{A}_{1} \times \mathcal{A}_{2}$ is an arrangement in $V_{1} \oplus V_{2}$ defined as in [6, Definition 2.13] by

$$
\mathcal{A}_{1} \times \mathcal{A}_{2}=\left\{H_{1} \oplus V_{2} \mid H_{1} \in \mathcal{A}_{1}\right\} \cup\left\{V_{1} \oplus H_{2} \mid H_{2} \in \mathcal{A}_{2}\right\} .
$$

Our main theorem is as follows:

Received by the editors May 16, 2008.

2000 Mathematics Subject Classification. Primary 32S22.

(C)2008 American Mathematical Society Reverts to public domain 28 years from publication 
Theorem 1.2. An arrangement $\mathcal{A}$ is totally free if and only if it has a decomposition

$$
\mathcal{A}=\mathcal{A}_{1} \times \mathcal{A}_{2} \times \cdots \times \mathcal{A}_{s}
$$

where each $\mathcal{A}_{i}$ is an arrangement in $\mathbb{K}^{1}$ or $\mathbb{K}^{2}$.

Ziegler showed in [12, Corollary 7$]$ that $(\mathcal{A}, m)$ is a free multiarrangement whenever $\ell \leq 2$. Note that

$$
D\left(\mathcal{A}_{1} \times \mathcal{A}_{2}, m\right) \simeq S \cdot D\left(\mathcal{A}_{1},\left.m\right|_{\mathcal{A}_{1}}\right) \oplus S \cdot D\left(\mathcal{A}_{2},\left.m\right|_{\mathcal{A}_{2}}\right)
$$

holds true as shown in [3, Lemma 1.4]. Thus

$$
\mathcal{A}_{1} \times \mathcal{A}_{2} \times \cdots \times \mathcal{A}_{s}
$$

is known to be totally free if each $\mathcal{A}_{i}$ is an arrangement in $\mathbb{K}^{1}$ or $\mathbb{K}^{2}$. Theorem 1.2 asserts that the converse is also true. In the next section we will prove Theorem 1.2 in a stronger form: we will show that $\mathcal{A}$ is decomposed into one-dimensional arrangements and two-dimensional ones if $\mathcal{N} \mathcal{F} \mathcal{M}(\mathcal{A})$ is a finite set.

Recall that the intersection lattice $L(\mathcal{A})$ is the set $\left\{X=H_{1} \cap \cdots \cap H_{s} \mid H_{i} \in\right.$ $\mathcal{A}, s \geq 0\}$ with the reverse inclusion ordering as in [6, Definition 2.1]. Then Theorem 1.2 implies:

Corollary 1.3. Whether an arrangement $\mathcal{A}$ is totally free or not depends only on its intersection lattice $L(\mathcal{A})$.

Let $\mathcal{A}$ be a nonempty central arrangement and $H_{0} \in \mathcal{A}$. Define the deletion $\mathcal{A}^{\prime}$ and the restriction $\mathcal{A}^{\prime \prime}$ as in [6, Definition 1.14]:

$$
\mathcal{A}^{\prime}:=\mathcal{A} \backslash\left\{H_{0}\right\}, \quad \mathcal{A}^{\prime \prime}:=\left\{H_{0} \cap H \mid H \in \mathcal{A}^{\prime}\right\} .
$$

Because of the characterization in Theorem 1.2, the total freeness is stable under deletion and restriction:

Corollary 1.4. Any subarrangement or restriction of a totally free arrangement is also totally free.

A multiarrangement was introduced and studied by Ziegler in [12. The third author proved in 9] and [10, that the freeness of a simple arrangement is closely related with the freeness of Ziegler's canonical restriction. Recently the first and second authors and Wakefield developed a general theory of free multiarrangements and introduced the concept of free multiplicity in [3] and [4. Several papers, including [1, 2], 5] and [1], studied the set of free multiplicities for a fixed arrangement $\mathcal{A}$. The main theorem (Theorem 1.2) in this article shows that the set of free multiplicities (or $\mathcal{N F \mathcal { F }}(\mathcal{A})$ ) imposes strong restrictions on the original arrangement $\mathcal{A}$.

\section{Proof of Theorem 1.2}

First we review in Theorem 2.1 a necessary condition for a given multiarrangement to be free.

Let $(\mathcal{A}, m)$ be a multiarrangement. When $(\mathcal{A}, m)$ is free, there exists a homogeneous basis $\theta_{1}, \ldots, \theta_{\ell}$ for $D(\mathcal{A}, m)$. The set $\exp (\mathcal{A}, m)$ of exponents is defined by $\exp (\mathcal{A}, m):=\left(\operatorname{deg} \theta_{1}, \ldots, \operatorname{deg} \theta_{\ell}\right)$, where $\operatorname{deg}\left(\theta_{i}\right):=\operatorname{deg} \theta_{i}(\alpha)$ for some linear form $\alpha$ with $\theta_{i}(\alpha) \neq 0$.

Define $L(\mathcal{A})_{2}:=\left\{X \in L(\mathcal{A}) \mid \operatorname{codim}_{V}(X)=2\right\}$ and $\mathcal{A}_{X}:=\{H \in \mathcal{A} \mid X \subset$ $H\}$. For $X \in L(\mathcal{A})_{2}$ the multiarrangement $\left(\mathcal{A}_{X},\left.m\right|_{\mathcal{A}_{X}}\right)$ is free with exponents 
$\left(d_{1}^{X}, d_{2}^{X}, 0, \ldots, 0\right)$. Define the second local mixed product $L M P_{2}(\mathcal{A}, m)$ as in 3 , Definition 4.3] by

$$
L M P_{2}(\mathcal{A}, m):=\sum_{X \in L(\mathcal{A})_{2}} d_{1}^{X} d_{2}^{X} .
$$

If $\mathcal{B}$ is a subarrangement of $\mathcal{A}$, then it is easy to see that

$$
L M P_{2}(\mathcal{A}, m) \geq L M P_{2}\left(\mathcal{B},\left.m\right|_{\mathcal{B}}\right) \text {. }
$$

Next assume that $(\mathcal{A}, m)$ is free with exponents $\left(d_{1}, \ldots, d_{\ell}\right)$. Define the second global mixed product $G M P_{2}(\mathcal{A}, m)$ as in [3, Definition 4.5] by

$$
G M P_{2}(\mathcal{A}, m):=\sum_{1 \leq i<j \leq \ell} d_{i} d_{j} .
$$

Theorem 2.1. If a multiarrangement $(\mathcal{A}, m)$ is free, then $\operatorname{GMP}_{2}(\mathcal{A}, m)=$ $L M P_{2}(\mathcal{A}, m)$.

In fact, Theorem 2.1 is true for any $G M P_{k}$ and $L M P_{k}(1 \leq k \leq \ell)$; see 3 , Corollary 4.6].

An arrangement $\mathcal{A}$ is said to be reducible if $\mathcal{A}=\mathcal{A}_{1} \times \mathcal{A}_{2}$ for certain arrangements $\mathcal{A}_{i}$ in $V_{i}(i=1,2)$. We say $\mathcal{A}$ is irreducible if it is not reducible.

Lemma 2.2. Let $\mathcal{A}$ be an irreducible arrangement in $\mathbb{K}^{\ell}$ with $\ell \geq 3$. Then there exists a subarrangement $\mathcal{B}$ with $|\mathcal{B}|=\ell+1$ such that:

$$
\operatorname{codim}_{V} H_{i} \cap H_{j} \cap H_{k}=3
$$

whenever $H_{i}, H_{j}, H_{k}$ are three hyperplanes in $\mathcal{B}$. Moreover the arrangement $\mathcal{B}$ is not free.

Proof. Let $H_{0} \in \mathcal{A}$. Let $\mathcal{A}^{\prime}$ and $\mathcal{A}^{\prime \prime}$ be the deletion and the restriction, respectively. Then either $\mathcal{A}^{\prime}$ or $\mathcal{A}^{\prime \prime}$ is irreducible by Tutte [8] (see also [7, Theorem 4.3.1]). When $|\mathcal{A}|=\ell+1$, the arrangement $\mathcal{A}$ itself satisfies the condition. We will prove by an induction on $|\mathcal{A}|$. If $\mathcal{A}^{\prime}$ is irreducible, then $\mathcal{A}^{\prime}$ contains $\ell+1$ hyperplanes satisfying the condition. So we may assume that $\mathcal{A}^{\prime \prime}$ is irreducible. Let $\mathcal{A}^{\prime}=$ $\left\{H_{1}, H_{2}, \ldots, H_{n-1}\right\}$ and $\overline{H_{i}}:=H_{i} \cap H_{0}$ for $1 \leq i \leq n-1$.

Suppose $\ell=3$. Since $\mathcal{A}^{\prime \prime}$ is irreducible, $3 \leq\left|\mathcal{A}^{\prime \prime}\right| \leq\left|\mathcal{A}^{\prime}\right|$.

Case 1. If $3 \leq\left|\mathcal{A}^{\prime \prime}\right|=\left|\mathcal{A}^{\prime}\right|$, then $\overline{H_{1}}, \overline{H_{2}}, \ldots, \overline{H_{n-1}}$ are distinct. Note that $H_{1} \cap H_{2} \cap$ $\cdots \cap H_{n-1}=\{\mathbf{0}\}$ because $\mathcal{A}$ is irreducible. Thus we may assume $H_{1} \cap H_{2} \cap H_{3}=\{\mathbf{0}\}$. So $\left\{H_{0}, H_{1}, H_{2}, H_{3}\right\}$ satisfies the condition.

Case 2. If $3 \leq\left|\mathcal{A}^{\prime \prime}\right|<\left|\mathcal{A}^{\prime}\right|$, then we may assume that $\overline{H_{1}}, \overline{H_{2}}, \overline{H_{3}}$ are distinct and $\overline{H_{3}}=\overline{H_{4}}$. Note that $H_{3} \cap H_{4} \subset H_{0}$. Thus

$$
H_{1} \cap H_{2} \cap H_{3} \cap H_{4}=\overline{H_{1}} \cap \overline{H_{2}} \cap \overline{H_{3}}=\{\mathbf{0}\} .
$$

Therefore either $H_{1} \cap H_{2} \cap H_{3}=\{\mathbf{0}\}$ or $H_{1} \cap H_{2} \cap H_{4}=\{\mathbf{0}\}$. So we may conclude that either $\left\{H_{0}, H_{1}, H_{2}, H_{3}\right\}$ or $\left\{H_{0}, H_{1}, H_{2}, H_{4}\right\}$ satisfies the condition.

Suppose $\ell \geq 4$. Then, by the induction assumption, there exists a subarrangement $\left\{\overline{H_{1}}, \overline{H_{2}}, \ldots, \overline{H_{\ell}}\right\}$ of $\mathcal{A}^{\prime \prime}$ satisfying the condition. Then the subarrangement $\left\{H_{0}, H_{1}, \ldots, H_{\ell}\right\}$ of $\mathcal{A}$ satisfies the condition. 
Suppose that the arrangement $\mathcal{B}$ is free. Then the sum of the exponents is equal to $\ell+1$. Thus one has $G M P_{2} \leq\left(\begin{array}{l}\ell \\ 2\end{array}\right)((\ell+1) / \ell)^{2}$. We also have $L M P_{2}=\left(\begin{array}{c}\ell+1 \\ 2\end{array}\right)$. Since

$$
L M P_{2}-G M P_{2} \geq\left(\begin{array}{c}
\ell+1 \\
2
\end{array}\right)-\left(\begin{array}{l}
\ell \\
2
\end{array}\right)((\ell+1) / \ell)^{2}=(\ell+1) / 2 \ell>0
$$

this contradicts Theorem 2.1

Recall

$$
\mathcal{N F \mathcal { M }}(\mathcal{A})=\left\{m: \mathcal{A} \rightarrow \mathbb{Z}_{>0} \mid m \text { is not a free multiplicity }\right\}
$$

Proposition 2.3. If $\mathcal{A}$ is an irreducible arrangement in $\mathbb{K}^{\ell}$ with $\ell \geq 3$, then $\mathcal{N F \mathcal { M }}(\mathcal{A})$ is an infinite set.

Proof. Suppose that $\mathcal{N} \mathcal{F M}(\mathcal{A})$ is a finite set. Choose a subarrangement $\mathcal{B}$ of $\mathcal{A}$ with $|\mathcal{B}|=\ell+1$ satisfying the condition in Lemma 2.2. Consider the multiplicity $m$ defined by

$$
m(H)= \begin{cases}1 & \text { if } H \notin \mathcal{B} \\ k & \text { if } H \in \mathcal{B}\end{cases}
$$

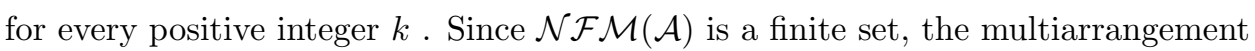
$(\mathcal{A}, m)$ is free whenever $k$ is sufficiently large. Note that $\left|L(\mathcal{B})_{2}\right|=\left(\begin{array}{c}\ell+1 \\ 2\end{array}\right)$. By the definition of $L M P_{2}$,

$$
L M P_{2}(\mathcal{A}, m) \geq L M P_{2}\left(\mathcal{B},\left.m\right|_{\mathcal{B}}\right)=\left|L(\mathcal{B})_{2}\right| k^{2}=\left(\begin{array}{c}
\ell+1 \\
2
\end{array}\right) k^{2} .
$$

Let $|\mathcal{A}|=n$. Then

$$
\sum_{d \in \exp (\mathcal{A}, m)} d=(k-1)(\ell+1)+n
$$

and thus

$$
G M P_{2}(\mathcal{A}, m) \leq\left(\begin{array}{l}
\ell \\
2
\end{array}\right)\left\{\frac{(k-1)(\ell+1)+n}{\ell}\right\}^{2}=\frac{(\ell+1)^{2}(\ell-1)}{2 \ell} k^{2}+A k+B
$$

with some constants $A$ and $B$. By Theorem 2.1 we have

$$
\left(\begin{array}{c}
\ell+1 \\
2
\end{array}\right) k^{2} \leq L M P_{2}(\mathcal{A}, m)=G M P_{2}(\mathcal{A}, m) \leq \frac{(\ell+1)^{2}(\ell-1)}{2 \ell} k^{2}+A k+B
$$

whenever $k$ is sufficiently large. This is a contradiction because

$$
\left(\begin{array}{c}
\ell+1 \\
2
\end{array}\right)-\frac{(\ell+1)^{2}(\ell-1)}{2 \ell}=\frac{\ell+1}{2 \ell}>0 .
$$

We now prove the following theorem, which is stronger than Theorem 1.2 .

Theorem 2.4. The following four conditions for a central arrangement $\mathcal{A}$ are equivalent:

(1) $\mathcal{A}$ is totally free; i.e., $\mathcal{N} \mathcal{F} \mathcal{M}(\mathcal{A})$ is empty.

(2) $\mathcal{N} \mathcal{F} \mathcal{M}(\mathcal{A})$ is a finite set. 


\section{(3) $\mathcal{A}$ has a decomposition}

$$
\mathcal{A}=\mathcal{A}_{1} \times \mathcal{A}_{2} \times \cdots \times \mathcal{A}_{s}
$$

where each $\mathcal{A}_{i}$ is an arrangement in $\mathbb{K}^{1}$ or $\mathbb{K}^{2}$.

(4) Every subarrangement of $\mathcal{A}$ is free.

Proof. The implications $(1) \Rightarrow(2),(3) \Rightarrow(4)$ and $(3) \Rightarrow(1)$ are obvious. Thus it is enough to prove that $(2) \Rightarrow(3)$ and $(4) \Rightarrow(3)$.

$(2) \Rightarrow(3)$ : Suppose that $\mathcal{N} \mathcal{F} \mathcal{M}(\mathcal{A})$ is a finite set. Decompose $\mathcal{A}$ into

$$
\mathcal{A}_{1} \times \mathcal{A}_{2} \times \cdots \times \mathcal{A}_{s}
$$

such that each $\mathcal{A}_{i}$ is irreducible. Since

$$
D(\mathcal{A}, m) \simeq S \cdot D\left(\mathcal{A}_{1},\left.m\right|_{\mathcal{A}_{1}}\right) \oplus S \cdot D\left(\mathcal{A}_{2},\left.m\right|_{\mathcal{A}_{2}}\right) \oplus \cdots \oplus S \cdot D\left(\mathcal{A}_{s},\left.m\right|_{\mathcal{A}_{s}}\right)
$$

holds by [3, Lemma 1.4], each $\mathcal{A}_{i}$ is an irreducible arrangement and $\mathcal{N} \mathcal{F} \mathcal{M}\left(\mathcal{A}_{i}\right)$ is a finite set. Thus Proposition 2.3 shows that each arrangement $\mathcal{A}_{i}$ is in $\mathbb{K}^{1}$ or $\mathbb{K}^{2}$.

$(4) \Rightarrow(3)$ : Decompose $\mathcal{A}$ into irreducible arrangements. Then each of the irreducible arrangements satisfies the assumption (4). Therefore we may assume that $\mathcal{A}$ is irreducible from the beginning. Then, by Lemma 2.2, we may conclude $\ell \leq 2$.

\section{ACKNOWLEDGEMENTS}

The first author is supported by the JSPS Research Fellowship for Young Scientists. The second and third authors have been supported in part by the Japan Society for the Promotion of Science. The authors thank Professors Sergey Yuzvinsky and Max Wakefield for helpful discussions and comments. We also thank Professor Thomas Zaslavsky for pointing out an error in an earlier version.

\section{REFERENCES}

[1] T. Abe, Free and non-free multiplicity on the deleted $A_{3}$ arrangement. Proc. Japan Acad. Ser. A 83 (2007), no. 7, 99-103. MR2361419

[2] T. Abe, K. Nuida and Y. Numata, Bicolor-eliminable graphs and free multiplicities on the braid arrangement. arXiv:0712.4110.

[3] T. Abe, H. Terao and M. Wakefield, The characteristic polynomial of a multiarrangement. Adv. in Math. 215 (2007), 825-838. MR2355609

[4] T. Abe, H. Terao and M. Wakefield, The Euler multiplicity and addition-deletion theorems for multiarrangements. J. London Math. Soc. 77 (2008), no. 2, 335-348. MR2400395

[5] T. Abe and M. Yoshinaga, Coxeter multiarrangements with quasi-constant multiplicities. arXiv:0708.3228.

[6] P. Orlik and H. Terao, Arrangements of hyperplanes. Grundlehren der Mathematischen Wissenschaften, 300. Springer-Verlag, Berlin, 1992. MR.1217488 (94e:52014)

[7] J. G. Oxley, Matroid Theory. Oxford University Press, New York, 1992. MR 1207587 (94d:05033)

[8] W. T. Tutte, Lectures on matroids. J. Res. Nat. Bur. Standards Sect. B 69B (1965), 1-47. MR0179781 (31:4023)

[9] M. Yoshinaga, Characterization of a free arrangement and conjecture of Edelman and Reiner. Invent. Math. 157 (2004), no. 2, 449-454. MR2077250 (2005d:52044)

[10] M. Yoshinaga, On the freeness of 3-arrangements. Bull. London. Math. Soc. 37 (2005), no. 1, 126-134. MR2105827 (2005i:52030) 
[11] M. Yoshinaga, On the extendability of free multiarrangements. arXiv:0710.5044.

[12] G. M. Ziegler, Multiarrangements of hyperplanes and their freeness. Singularities (Iowa City, IA, 1986), 345-359, Contemp. Math., 90, Amer. Math. Soc., Providence, RI, 1989. MR.1000610 (90e:32015)

Department of Mathematics, Hokkaido University, Kita-10, Nishi-8, Kita-Ku, SAPPORO, 060-0810, JAPAN

Current address: Department of Mathematics, Kyoto University, Kitashirakawa-Oiwake-Cho, Sakyo-Ku, Kyoto, 606-8502, Japan

E-mail address: abetaku@math.kyoto-u.ac.jp

Department of Mathematics, Hokkaido University, Kita-10, Nishi-8, Kita-Ku, SAPPORO, 060-0810, JAPAN

E-mail address: terao@math.sci.hokudai.ac.jp

Department of Mathematics, Kobe University, 1-1 Rokkodai, Nada-ku, Kobe, 6578501, JAPAN

E-mail address: myoshina@math.kobe-u.ac.jp 